ARTÍCULOS ORIGINALES

Rev Chil Salud Pública 2018; Vol 22 (1): 41-50
CARIES DENTAL, ENFERMEDAD

PERIODONTAL Y CONOCIMIENTOS EN HIGIENE BUCAL POST ALTA EN PACIENTES GES EMBARAZADA DE UN CENTRO DE SALUD FAMILIAR DE CHILLÁN

\author{
DENTAL CAVITIES, PERIODONTAL DISEASE, AND ORAL HYGIENE \\ KNOWLEDGE OF POST-DISCHARGE EXPLICIT HEALTH GUARANTEES \\ PREGNANT PATIENTS IN A FAMILY HEALTH CENTER IN CHILLÁN
}

\title{
RESUMEN
}

Las enfermedades bucales de mayor prevalencia en Chile son la caries y la enfermedad periodontal, y las mujeres embarazadas no son la excepción. Este trabajo se refiere a este caso.

Objetivo: Evaluar el efecto de la intervención odontológica en el número de caries dental y estado de salud periodontal post alta integral de las usuarias Programa Odontológico GES Embarazada año 2013 del CESFAM Violeta Parra de Chillán, Chile.

Material y métodos: Se realizó un estudio analitico de corte transversal en 67 embarazadas de alta integral en 2013 y evaluadas en box dental en 2016. Como fuente de información se utilizaron: Examen clinico, anamnesis odontológica, fichas clinicas, encuesta de hábitos de higiene bucal. El estudio fue autorizado por el comité de ética acreditado del hospital Herminda Martín de Chillán. Se utilizó estadística descriptiva e inferencial empleando el programa SPSS con un valor de a de 0,05.

Resultados: Las caries dentales disminuyeron significativamente de 10,34 a 0,81 post intervención ( $p<0,0001$ ). Un 97\% de las usuarias presentó un estado de salud periodontal malo o regular en 2013, el que mejoró ostensible en 2016 con solo un 4,5\% en esta categoría ( $p<0,0001)$. Respecto a bábitos de higiene bucal, más del $95 \%$ de las participantes realizó una correcta técnica de cepillado y consideró que el cepillado de dientes es la berramienta más importante para la prevención de las caries.

Conclusión: La intervención odontológica realizada mediante el Programa GES Embarazada disminuye significativamente la presencia de caries post alta integral. La salud periodontal de las usuarias mejora y se

María Jesús Salazar

Cirujano Dentista, Magíster en Salud Pública Universidad del Bío-Bío. majesussalazar@gmail.com

\section{Julio Parra}

Magíster en Salud Pública,

Doctor en Ciencia

de los Alimentos,

Universidad del Bío-Bío
Palabras clave: caries dental, enfermedad periodontal, intervención odontológica, Programa GES embarazada.

La investigación formó parte de la tesis de grado de María Jesús Salazar Soto para optar al grado de Magíster en Salud Pública cuyo docente guía fue el Dr. Julio Parra.

No se declaran conflictos de interés.

Trabajo recibido el 3 de junio de 2017. Aceptado a publicación el 17 de noviembre de 2017 


\section{ABSTRACT}

The most prevalent oral diseases in Chile are dental cavities and periodontal disease, and pregnant women are not the exception. This study investigates this population.

Objective: To evaluate the effect of dental interventions on the number of dental cavities and the periodontal health status of post-discharge participants of the 2013 GES Dental Program for Pregnant Women in the Violeta Parra CESFAM (primary care center) in Chillan, Chile.

Materials and methods: An analytical cross-sectional study was conducted with 67 pregnant women discharged in 2013 and evaluated in a dental check-up in 2016. Sources of information were: clinical examination, dental anamnesis, clinical records, and a survey of oral bygiene habits. The study was authorized by the Ethics Committee of the Chillan Herminda Martin Hospital. Descriptive and inferential statistics were used with the SPSS program, with $\alpha=0.05$.

Results: Dental cavities significantly decreased from 10.34 to 0.81 post intervention ( $p<$ 0.0001). While $97 \%$ of participants exhibited bad or fair periodontal health in 2013 , this variable noticeably improved in 2016, with only $4.5 \%$ of users in this category $(p<0.0001)$. Regarding oral bygiene habits, participants used a correct brushing technique, and they considered toothbrushing to be the most important tool to prevent cavities.

Conclusion: The dental intervention performed by the GES Program for Pregnant Women significantly decreased the number of post-discharge dental cavities. Periodontal health of the participants improved and was maintained three years after discharge from the program.

Keywords: dental cavities, periodontal disease, dental intervention, GES program for pregnant women.

\section{INTRODUCCIÓN}

La caries dental y la enfermedad periodontal son las enfermedades bucales más prevalentes a nivel mundial. ${ }^{1}$ En Chile estas enfermedades son las principales causas de pérdida de piezas dentales en la población, donde cerca del $100 \%$ de los adultos presentan caries dental y un $15 \%-20 \%$ de ellos presenta enfermedad periodontal., ${ }^{2,3,5}$ Además, la población de 35 a 44 años tiene un promedio de 6,5 dientes perdidos respecto de la población de 65 a 74 años con 15,8 dientes. ${ }^{6}$ Las cifras manifiestan un problema real en la población afectando la calidad de vida y salud. ${ }^{7,8}$ Por ello el Ministerio de Salud de Chile el año 2006 incorpora a la política de Garantías Explícitas en Salud (GES) el Programa Odontológico, el cual consiste en un conjunto de estrategias que dan respuesta a los problemas de salud bucal de niños y niñas de 6 años, embarazadas, adultos de 60 años y atención de urgencias odontológicas dirigidas a los beneficiarios del sistema público de salud. ${ }^{9}$

Respecto a la salud bucal de la mujer embarazada, la Organización Mundial de la Salud (OMS) y el Ministerio de Salud de Chile
(MINSAL) consideran clave el enfoque de la salud en el curso de la vida, mediante la prevención y control de las enfermedades crónicas no transmisibles. Esto implica intervenir durante todo el ciclo de la vida, comenzando con la salud de la madre, incluyendo la etapa preconcepcional, prenatal y postnatal, siguiendo posteriormente con la infancia y la adolescencia. ${ }^{10}$ Es por ello que para muchos países la atención odontológica a las embarazadas es prioritaria en sus sistemas de salud. Estados Unidos el año 2006 señaló que un $25 \%$ de las mujeres norteamericanas en edad reproductiva habían tenido caries y entre un 37 y $46 \%$ de las mismas presentaron enfermedad periodontal. Esta última se presentó además sobre el $30 \%$ de las mujeres embarazadas del mismo país. Sin embargo, a pesar de que el sistema de salud norteamericano ha garantizado el acceso a la atención odontológica integral para las embarazadas, esta solo es entregada a quienes cuentan con seguro médico. Por consiguiente, para muchas de las mujeres norteamericanas el embarazo es la única oportunidad que disponen para acceder a atención odontológica integral, ya que terminado este período normalmente pierden la 
cobertura odontológica; hecho relacionado a las condiciones que les ofrece el seguro que pueden pagar. ${ }^{11}$

En Chile, el MINSAL reconoció la importancia de la gestación tanto para la mujer como para su hijo y la familia, priorizando su atención odontológica; generando las condiciones para que el recién nacido crezca en un entorno familiar que promueva y proteja su salud bucal. ${ }^{12,13}$ Durante el embarazo, los cambios hormonales, los hábitos alimentarios y los requerimientos nutricionales provocan también cambios en la salud oral de las mujeres, propiciando la aparición de caries y enfermedad periodontal. ${ }^{14,15}$ Además, existe evidencia respecto a la asociación de la enfermedad periodontal con la presencia de parto prematuro; de la misma forma se relaciona esta enfermedad en madres de niños que tuvieron bajo peso al nacer. ${ }^{16}$ El GES Odontológico de la Embarazada consiste en una atención integral realizada por un cirujano dentista, según las necesidades de la paciente, dirigida a recuperar y rehabilitar la salud bucal de la mujer gestante así como también educarla en higiene bucal para prevenir y mantener este tratamiento en el tiempo. ${ }^{17}$ Todo esto con la garantía de que el tratamiento será realizado oportunamente y de manera gratuita para quienes pertenecen al Fondo Nacional de Salud grupos A y B, mientras que los grupos $\mathrm{C}$ y D y quienes pertenecen a ISAPRE deberán pagar como máximo un $20 \%$ del costo total de todo el tratamiento. ${ }^{18,19}$ Este programa es una intervención de alto impacto, ya que constituye una oportunidad única para modificar conductas que se han asociado a un riesgo mayor de problemas de salud..$^{20}$ Sin embargo, la evaluación de los resultados del Programa GES Embarazada se remite al número de altas obtenido en el año calendario. ${ }^{9}$ Hasta la fecha no se han encontrado estudios publicados en Chile que aborden el tema de la salud bucal post alta de las usuarias del Programa GES Embarazada, en lo que respecta a la mantención en el tiempo del tratamiento recibido, así como tampoco si han continuado con el cuidado de su salud bucal e higiene y la de sus hijos.

Por lo anterior se decidió evaluar la presencia de caries dental, enfermedad perio- dontal y conocimientos en higiene bucal en usuarias del Programa GES Embarazada, 3 años post intervención en el CESFAM Violeta Parra de Chillán.

\section{MATERIAL Y MÉTODO}

La presente investigación corresponde a un estudio analítico de corte transversal en un universo censado de 67 usuarias del Programa GES Embarazada del CESFAM Violeta Parra en el año 2013. Como criterios de elegibilidad se consideró a pacientes inscritas en el CESFAM, tener alta integral odontológica el año 2013 y no haber recibido ningún tipo de atención odontológica posterior a ese año.

Previo a la investigación se revisó la ficha dental de las usuarias atendidas el año 2013 para determinar el número de caries y estado de salud periodontal que presentaron con anterioridad a la intervención. Con posterioridad, mediante examen clínico, se determinó la presencia actual en 2016 de caries dental y enfermedad periodontal. Además, se les consultó sobre sus hábitos de higiene bucal, registrando en una encuesta los aspectos contenidos en la intervención odontológica consistentes en: frecuencia de cepillado, técnica de cepillado dental, recambio de cepillo dental, uso de seda dental, uso de colutorio (enjuague bucal), cepillado de dientes de su hijo(a), cepillado de dientes frente a sus hijos e importancia del cepillado dental para sus hijos.

Para recolectar la información se confeccionó una ficha en la cual se registraron los datos de caracterización de la muestra (edad, estado civil y ocupación), el número de caries y el índice de Green y Vermillion (mediante el cual se determinó el estado de salud periodontal en las usuarias). En el examen clínico se utilizó sonda curva, espejo y pinza de acuerdo a los parámetros de diagnóstico de la OMS. ${ }^{21}$ Para conocer sus hábitos de higiene bucal, se utilizó una encuesta, la que fue validada previamente en un grupo piloto de embarazadas del CESFAM Violeta Parra pertenecientes al Programa del año 2016 que consideraba los aspectos contenidos en la intervención odontológica normada según el MINSAL. 
Antes de comenzar el estudio se realizó una recopilación de los datos de las usuarias y posteriormente se les citó para explicarles el motivo de la investigación y preguntarles acerca de su interés por participar en él. La recolección de los datos se realizó en la jornada vespertina del CESFAM, en un período de 3 meses desde octubre a diciembre de 2016. El estudio fue previamente aprobado por el comité de ética del Hospital Clínico Herminda Martín de Chillán con el registro $n^{\circ} 276$ y además se firmó un consentimiento informado por parte de todos las participantes del estudio respecto a la utilización de la información y el resguardo de su identidad, ajustándonos a la normativa existente en Chile sobre investigación y confidencialidad de los datos de los participantes en una investigación científica. ${ }^{22}$

En el análisis se utilizó estadística descriptiva, y para conocer la distribución normal de los datos se aplicó la prueba de Shapiro-Wilk. Respecto a la relación entre el número de caries pre y post intervención odontológica, se aplicó la prueba de T-Student para muestras pareadas. Finalmente, para relacionar el estado de salud periodontal entre los grupos muy bueno-bueno y regular-mala, pre y post intervención odontológica, se utilizó la prueba exacta de Fisher con un nivel de significancia de 0,05. Para todo lo anterior se trabajó con el programa SPSS versión 21.0 para Windows, con licencia registrada.

\section{RESULTADOS}

El grupo de estudio quedó conformado por mujeres con una edad promedio de 30 años $(\mathrm{DS} \pm 6,03)$, solteras en un $61 \%$ y mayoritariamente trabajadoras dependientes, $56,7 \%$.
En la Tabla 1 se observa que luego de la revisión clínica de las participantes en el año 2013, las usuarias presentaron previo a la intervención odontológica una mediana de 10,34 caries, mientras que al ser examinadas post alta integral odontológica en el año 2016 presentaron una reducción significativa con 0,81 caries dental. En la Tabla 2 para la salud periodontal, se muestra que antes de comenzar su tratamiento el año 2013, un $97 \%$ de las usuarias presentó un estado de salud malo o regular, el que mejoró ostensiblemente en 2016, ya que se encontró esta categoría solo en un $4,5 \%$.

La Tabla 3 muestra, mediante el autorreporte de las participantes del estudio en el 2016, los conocimientos de higiene bucal, componente principal de la intervención odontológica realizada. Se pudo determinar que la frecuencia promedio de cepillado diario dental es de 3 veces al día. Asimismo, el recambio promedio del cepillo de dientes es cada 3 meses, con una correcta técnica de cepillado en un $95,5 \%$ de las usuarias. Finalmente, un $97 \%$ reconoció que una correcta técnica de cepillado es la herramienta más importante para prevenir la caries. Respecto a los complementos de higiene bucal, un 46,4\% de las usuarias reconoció usar seda dental, mientras que solo un $22,4 \%$ utiliza enjuague bucal. Las últimas preguntas de la encuesta dieron como resultado que $97 \%$ de las usuarias afirma que cepilla los dientes de sus hijos de 3 años e incluso un $83 \%$ de ellas se cepilla los dientes frente a sus hijos para motivarlos a adoptar hábitos de higiene bucal. A ello se suma que el $100 \%$ de las usuarias enseñan a sus hijos sobre lo importante del cepillado dental para mantener sus dientes sanos.

Tabla 1. Número de caries en el grupo de estudio pre y post intervención odontológica

\begin{tabular}{|c|c|c|c|c|c|}
\hline Caries & $\mathbf{n}$ & Mínimo & Mediana & Máximo & $\begin{array}{c}\text { IC 95\% } \\
\text { Linf - Lsup }\end{array}$ \\
\hline 2013 & 67 & 2 & 10,34 & 17 & $9,214-11,466$ \\
\hline 2016 & 67 & 0 & 0,81 & 6 & $0,473-1,147$ \\
\hline
\end{tabular}


Tabla 2. Estado de salud periodontal de las participantes del estudio pre y post intervención odontológica

\begin{tabular}{|c|c|c|}
\hline Estado de Salud Periodontal & $\begin{array}{c}\text { 2013 } \\
\mathbf{n}(\%) \text { IC 95\% } \\
\text { Linf - Lsup }\end{array}$ & $\begin{array}{c}\text { 2016 } \\
\mathbf{n} \text { (\%) IC 95\% } \\
\text { Linf - Lsup }\end{array}$ \\
\hline Excelente & $0(0)-$ & $25(37,3) 26,70-49,30$ \\
\hline Buena & $2(3,0) 0,21-10,86$ & $39(58,2) 46,26-69,27$ \\
\hline Regular & $19(28,4) 18,91-40,15$ & $3(4,5) 1,03-12,87$ \\
\hline Malo & $46(68,6) 56,75-78,54$ & $0(0)$ \\
\hline Total & $67(100)$ & $67(100)$ \\
\hline
\end{tabular}

Tabla 3. Hábitos de higiene bucal de las participantes del estudio

\begin{tabular}{|c|c|c|c|}
\hline & $\mathbf{n}$ & Mediana & I.C 95\% \\
\hline Frecuencia de cepillado & 67 & 2,90 & $2,727-3,073$ \\
\hline Recambio del cepillo de dientes & 67 & 2,90 & $2,727-3,073$ \\
\hline
\end{tabular}

\begin{tabular}{|c|c|c|c|c|}
\hline & & $\mathrm{n}$ & $\%$ & I.C $95 \%$ \\
\hline \multirow{3}{*}{ Técnica de cepillado } & Correcta & 64 & $95,5 \%$ & $88,99-98,52$ \\
\hline & Incorrecta & 3 & $4,5 \%$ & $1,03-12,87$ \\
\hline & Total & 67 & $100 \%$ & \\
\hline \multirow{3}{*}{$\begin{array}{l}\text { Prevención de } \\
\text { caries mediante el } \\
\text { cepillado }\end{array}$} & Correcta & 65 & $97 \%$ & $89,14-99,79$ \\
\hline & Incorrecta & 2 & $3 \%$ & $0,21-10,86$ \\
\hline & Total & 67 & $100 \%$ & \\
\hline \multirow{3}{*}{$\begin{array}{l}\text { Uso de } \\
\text { seda dental }\end{array}$} & Sí & 31 & 46,3 & $34,86-58,09$ \\
\hline & No & 36 & 53,7 & $41,91-6514$ \\
\hline & Total & 67 & 100,0 & \\
\hline \multirow{3}{*}{$\begin{array}{l}\text { Uso de } \\
\text { colutorio }\end{array}$} & Sí & 15 & 22,4 & $13,96-33,81$ \\
\hline & No & 52 & 77,6 & $66,19-86,04$ \\
\hline & Total & 67 & 100,0 & \\
\hline \multirow{3}{*}{$\begin{array}{l}\text { Cepillado dental de } \\
\text { sus hijos }\end{array}$} & Sí & 65 & $97 \%$ & $89,14-99,79$ \\
\hline & No & 2 & $3,0 \%$ & $0,21-10,86$ \\
\hline & Total & 67 & $100 \%$ & \\
\hline \multirow{3}{*}{$\begin{array}{l}\text { Cepillado dental } \\
\text { frente a sus hijos }\end{array}$} & Sí & 56 & $83 \%$ & $72,77-90,75$ \\
\hline & No & 11 & $16 \%$ & $9,25-27,23$ \\
\hline & Total & 67 & $100 \%$ & \\
\hline $\begin{array}{l}\text { Importancia del } \\
\text { cepillado dental para } \\
\text { sus hijos }\end{array}$ & Sí & 67 & $100 \%$ & \\
\hline
\end{tabular}


Tabla 4. Variación en el número de caries durante pre y post intervención odontológica

\begin{tabular}{|l|l|l|l|}
\hline & $\begin{array}{l}\text { Caries 2013 } \\
\text { Mediana }\end{array}$ & $\begin{array}{l}\text { Caries 2016 } \\
\text { Mediana }\end{array}$ & Valor $\mathbf{p}$ \\
\hline No Caries & 10,34 & 0,81 & 0,0001 \\
\hline
\end{tabular}

P value: Prueba T de Student para muestras pareadas.

Tabla 5. Cambio en el estado de salud periodontal pre y post intervención odontológica

\begin{tabular}{|l|l|l|l|l|}
\hline \multicolumn{5}{|c|}{ Estado de Salud Periodontal } \\
\hline Año & $\mathbf{n}$ & Excelente - Bueno & Regular- Malo & Valor p \\
\hline 2013 & 67 & 2 & 65 & 0,0001 \\
\hline 2016 & 67 & 64 & 3 & \\
\hline
\end{tabular}

P value: Test exacto de Fisher.

En la Tabla 4, se observa que existen diferencias significativas entre el número de caries que fueron tratadas al ingreso del tratamiento el año 2013 comparadas con el número de caries presentadas con posterioridad al alta integral odontológica en 2016 ( $\mathrm{p}<0,05)$.

Finalmente, existe una disminución significativa (Tabla 5) al evaluar la intervención odontológica en la salud periodontal al comienzo del tratamiento y al control post alta luego de 3 años $(\mathrm{p}<0,05)$.

\section{DISCUSIÓN}

Las 67 usuarias que participaron de este estudio recibieron el año 2013 un tratamiento odontológico integral, siendo dadas de alta sin caries ni enfermedad periodontal. De este grupo el promedio de edad fue de 30 años, similar al estudio de Díaz-Romero y cols., respecto a la edad promedio de las embarazadas que consultan por atención odontológica. ${ }^{23}$

Los resultados previos a la intervención arrojaron una alta prevalencia de caries, similar a la publicada por Ruíz y cols., respecto de la prevalencia de caries en un grupo de embarazadas atendidas en un servicio de salud pública mexicano. ${ }^{24}$ Afortunadamente, la disminución en el número de caries post intervención odontológica indica que hubo un interés por mantener el tratamiento recibido por parte de las participantes del estudio. Aspecto muy similar informado por Carrión, que plantea que a lo largo de la última década ha existido un aumento en el interés por atención odontológica por parte de las mujeres embarazadas.

De igual forma ocurre al observar el estado de salud periodontal pre y post intervención odontológica, en donde es común observar al inicio del tratamiento un mal estado de salud periodontal, lo que es similar a lo publicado en otros países. Así lo afirma el estudio de Díaz-Guzmán y cols. que informan altos niveles de prevalencia de enfermedades periodontales en pacientes embarazadas atendidas en una escuela dental de México. ${ }^{26}$

Lo significativo de los resultados obtenidos radica en que luego de 3 años post alta integral, las usuarias mantienen buenos índices de salud periodontal, considerando que no recibieron ningún tipo de atención odontológica durante todo el período post alta.

A lo anterior se debe agregar que los resultados obtenidos en encuesta sobre higiene bucal señalan que las usuarias post alta integral odontológica mantienen correctos hábitos de higiene bucal respecto a la frecuencia y técnica de cepillado. Artázcos y cols. reportaron que la frecuencia de cepillado dental en adolescentes españoles fue de más de una vez al día, al igual que lo observado en este 
estudio, y con ello mantenían buenos niveles de higiene bucal, pero con irregular uso de enjuague bucal y seda dental. ${ }^{27}$ Esto se debe según González, a que los pacientes no utilizan los complementos del cepillado para una mejor higiene bucal porque los consideran innecesarios o bien no saben utilizarlos. ${ }^{28}$ Por otra parte, un gran porcentaje de las usuarias reconoce en el cepillado la mejor herramienta para la prevención de la caries, así como también casi la totalidad cepilla los dientes de sus hijos a diario, cepillándose ellas mismas frente a ellos como modelo de hábito y enseñándoles la importancia del cepillado para tener dientes sanos. Este aspecto fue planteado por Nunez y cols., quienes indicaron la necesidad de evaluar tanto la utilización del programa GES Embarazada como también el nivel de conocimiento de las madres sobre hábitos de higiene bucal y la entrega de estos conocimientos de autocuidado a su futuro hijo desde la aparición de sus primeros dientes como una estrategia efectiva de promoción de una adecuada salud bucal. ${ }^{29}$

Si consideramos que, de acuerdo al estudio de Pérez y cols., la aparición de caries y enfermedad periodontal se atribuye principalmente a la dieta cariogénica y la higiene bucal deficiente, resulta aún más importante valorar el componente educativo de la aplicación de este programa. En nuestro estudio no fue posible hacer un diagnóstico de conocimientos pre intervención odontológica, sin embargo, teniendo en cuenta que todas las participantes del estudio recibieron educación de su odontólogo tratante sobre el tema, podemos inferir la importancia de contar con nociones concretas de higiene bucal para la mantención en el tiempo del tratamiento recibido y lo importante que es traspasar estos hábitos a sus hijos y hacerlos responsables de su salud bucal.

Finalmente, los resultados obtenidos al relacionar la intervención odontológica sobre el número de caries y estado de salud periodontal pre y post alta integral permiten observar cambios significativos respecto al tratamiento odontológico entregado el año 2013 y la reevaluación en el $2016(\mathrm{p}<0,05)$. A pesar de que en la actualidad no existen investigacio- nes respecto a la mantención de tratamientos odontológicos post intervención odontológica en embarazadas, Montecinos mostró en su estudio en Chillán (manuscrito en revisión) que una intervención odontológica compuesta de actividades recuperativas y educación sobre hábitos de higiene bucal en escolares de 12 años logra cambios positivos en la disminución de la caries y la salud periodontal de los pacientes post alta integral. ${ }^{31}$

Si bien este tipo de estudio tiene ciertas limitantes respecto a la selección de la muestra ya que se trataba de una población pequeña, el grupo de Embarazadas logró ser evaluada en su totalidad. Otra limitante, es la posibilidad del sesgo de cortesía o de información en la encuesta de hábitos de higiene bucal, el cual no puede evitarse; sin embargo, los resultados obtenidos en el examen clínico en boca reflejan en cierta forma lo informado en la encuesta.

Finalmente, si bien este tipo de estudios no puede entregarnos una relación directa causa-efecto respecto a la intervención odontológica y la presencia de caries y enfermedad periodontal a lo largo del tiempo; los resultados obtenidos en esta investigación revelan una disminución significativa de ambas patologías en las pacientes atendidas luego de 3 años post alta y una conservación en el tiempo del tratamiento recibido. Esto entrega información relevante para el diseño de un nuevo estudio que permita evaluar el impacto de estas intervenciones en salud a lo largo de un período determinado. ${ }^{32}$

\section{CONCLUSIONES}

Después de tres años post alta integral del Programa Odontológico GES Embarazada, compuesto de actividades recuperativas y educación en salud bucal, es posible observar una disminución en el número de caries y enfermedad periodontal en las pacientes que recibieron este tratamiento.

Considerando lo anterior, es necesario controlar el tratamiento odontológico entregado post alta como una estrategia de conservación de los tratamientos dentales disponibles en el sistema público, ya que en 
teoría debería ser más conveniente en términos económicos controlar periódicamente y tratar una o dos piezas con caries que luego de muchos años volver a tratar por completo a una paciente. No debemos olvidar que los pacientes de programas especiales GES no acceden a control a mediano o largo plazo, solo tienen garantía de tratamiento por un año. Por lo tanto, en el caso de las usuarias del CESFAM Violeta Parra, si éstas llegaran a necesitar en el futuro atención odontológica, deberán adaptarse a lo que su CESFAM les ofrece para dar solución a sus problemas de salud dental, generalmente en las consultas de urgencia o jornadas vespertina mediante lista de espera.

Es necesario evaluar los costos de controlar a las usuarias comparado con volver a tratar a largo plazo, ya que el sistema de salud no ha publicado información respecto a la conveniencia de la mantención en el tiempo de los programas odontológicos GES como una forma de disminuir en el futuro los costos de un nuevo tratamiento completo.

No es posible que la única atención dental garantizada a las mujeres sea el programa GES Embarazada y luego de esto esperar hasta los 60 años para otra atención integral garantizada por ley. Por ello, es necesario crear políticas que garanticen acceso y oportunidad de atención a las mujeres de 20 a 59 años de edad.

Finalmente, y como una forma de sacar el máximo de provecho a todo lo que entrega el Programa Odontológico GES Embarazada, sería interesante conocer, junto con la salud bucal de las madres post alta integral, la salud bucal de sus hijos. Así lo plantea Córdova (2007), al afirmar que el Programa Odontológico GES Embarazada trasciende más allá de entregar salud bucal a las futuras madres, pues también resulta fundamental en la futura salud bucal de los niños, ya que éstos recibirán tempranamente hábitos de autocuidado en higiene bucal arraigándolos en sus quehaceres cotidianos y haciéndolos responsables de la salud de su dentadura. ${ }^{33}$

Por todo lo anterior, considerando los muy buenos resultados obtenidos en esta investigación, es necesario realizar un nuevo diseño de investigación epidemiológica que permita, por un lado, establecer que este tipo de intervención tiene un efecto significativo en la disminución de la caries y la enfermedad periodontal de las pacientes a lo largo del tiempo; así como también establecer si el componente educativo de este programa trasciende desde las madres a los hijos en lo que respecta a una baja prevalencia de caries y hábitos adquiridos de autocuidado en salud bucal.

Este tipo de estudio entregará información concreta que permita replantear el diseño de las actuales políticas en salud bucal, en pro de una salud pública más comprometida con el empoderamiento de sus usuarios, haciéndoles responsables de su salud y la de sus familias.

\section{REFERENCIAS}

1. Organización Mundial de la Salud. Salud Bucodental. Nota Informativa $\mathrm{N}^{\circ} 318 ; 2012$ (Abril). [consultado 22.10.2015]. Disponible en: http://www.who.int/mediacentre/factsheets/fs318/es/.

2. Chile. Ministerio de Salud. Subsecretaría de Salud Pública. División Prevención y Control de Enfermedades. Análisis de Situación Salud Bucal en Chile. Departamento de salud Bucal. Diciembre 2010. [consultado 25.10.2015]. Disponible en : http://web.minsal.cl/sites/default/files/files/An \% C3\% A1lisis \% 20de \% 20 Situaci\%C3\%B3n\%20Salud\%20Bucal\%20 final\%20pdf.pdf.

3. Arteaga O, Urzúa I, Espinoza I, Muñoz A, Mendoza C, Arteaga O, et al. Prevalencia de caries y pérdida de dientes en población de 65 a 74 años de Santiago, Chile. Rev Clin Periodoncia Implantol Rehabil Oral, 2009; 2(3): 161-166.

4. Angel P, Fresno MC, Cisternas P, Lagos M, Moncada G. Prevalencia de caries, pérdida de dientes y necesidad de tratamiento en población adulta mapuche-huilliche de Isla Huapi. Rev Clin Periodoncia Implantol Rehabil Oral , 2010; 3(2): 69-72.

5. Gamonal J, Mendoza C, Espinoza I, Muñoz A, Urzúa I, Aranda W, et al. Clinical attachment loss in Chilean adult population: First Chilean National Dental Examination Sur- 
vey. J Periodontol., 2010; 81(10): 1403-1410.

6. Urzúa I, Mendoza C, Arteaga O, Rodríguez G, Cabello R, Faleiros S, et al. Dental caries prevalence and tooth loss in chilean adult population: first national dental examination survey. Int J Dent., 2012; 810: 170.

7. Chile. Ministerio de Salud. División de Planificación Sanitaria. Estudio de Preferencias Sociales para la definición de Garantías Explícitas en Salud. Santiago, Chile: MINSAL; 2008 [consultado el 16.10.2015]. Disponible en: http://epi.minsal.cl/epi/html/invest/IF_ ESTUDIO_PREFERENCIAS_SOCIALES_GES_2009.pdf

8. Chile. Ministerio de Salud. Metas 20112020. Estrategia Nacional de Salud para el cumplimiento de los objetivos sanitarios de la década 2011-2020. Santiago, Chile: MINSAL; 2011 [consultado 25.10.2015]. Disponible en: http://www.minsal.cl/portal/url/ item/c4034eddbc96ca6de0400101640159b8. pdf

9. Chile. Ministerio de Salud. Subsecretaría de Redes Asistenciales. División Atención de Primaria. Departamento Odontológico. Aprueba Programa GES Odontológico. Santiago, Chile; 2013 [consultado 25.10.2015]. Disponible en: https://www.araucanianorte.cl/images/PDF-WORD/ Res-Ex-1217-Programa-GES-Odontologico-2015-APS-02122014.pdf

10. WHO. Global action plan for the prevention and control of noncommunicable diseases 2013-2020. Geneva, Switzerland, 2013; pp. 12-13. Disponible en: http://www.who.int/ $\mathrm{nmh} /$ events/2013/revised_draft_ncd_action_plan.pdf?ua=1

11. USA. New York State Department of Health. Oral health care during pregnan$c y$ and early childhood: practice guidelines. New York, USA: New York State Department of Health; 2006.

12. Chile. Ministerio de Salud. Guía clínica salud oral integral de la embarazada. Santiago, Chile: MINSAL; 2013.

13. Kloetzel MK, Huebner CE, Milgrom P. Referrals for dental care during pregnancy. $J$ Mid Womens Health, 2011; 56(2): 110-7.

14. American Academy of Pediatric Dentistry. Guideline on Perinatal Oral Health
Care. Reference Manual, 2011; 37(6): 15-16 [consultado noviembre 2015]; Disponible en: http://www.aapd.org/media/Policies_ Guidelines/G_PerinatalOralHealthCare. pdf.

15. Silk H, Douglass AB, Douglass JM, Silk L. Oral health during pregnancy. Am Fam Physician., 2008; 77(8): 1139-44.

16. Gibbs RS. The relationship between infections and adverse pregnancy outcomes: an overview. Annals of Periodontology. J Periodontol., 2001; 6(1): 153-63.

17. Boggess KA, Edelstein BL. Oral health in women during preconception and pregnancy: implications for birth outcomes and infant oral health. Matern Child Health J., 2006; 10(5): 69-174.

18. Chile. Superintendencia de Salud. Circular IF $N^{\circ}$ 122: Decreto Supremo $N^{\circ} 1$, de los Ministerios de Salud y de Hacienda, que aprueba las Garantías Explícitas en Salud y fija texto refundido. Santiago, Chile, 30 de junio de 2010 [consultado abril 2016]. Disponible en: http://www.supersalud.gob.cl/normativa/668/articles-6029_recurso_1.pdf

19. Chile. Ministerio de Salud. Aprueba garantías explícitas en salud del Régimen general de garantías en salud.Chile: MINSAL; 2013 [consultado 27.06.2015]. Disponible en: http://www.minsal.gob.cl/portal/url/item/ d692c627c623b9cae040010164016563.pdf.

20. Chile. Ministerio de Salud. Subsecretaría de Salud Pública. Departamento de Salud Bucal. Orientación técnica para la educación en salud bucal de la embarazada. Santiago, Chile: MINSAL; 2013.

21. World Health Organization. Oral health surveys: basic methods (4rd ed). Suiza: WHO; 1997 [consultado 26.04.2016]. Disponible en: http://new.paho.org/hq/dmdocuments/2009/OH_st_Esurv.pdf

22. Ley $\mathrm{N}^{\circ} 20.584$. Regula los derechos y deberes que tienen las personas en relación con acciones vinculadas a su atención en salud. Diario Oficial la República de Chile (del 24 de octubre de 2012) [consultado marzo 2016]. Disponible en: http://www.bcn.cl/ leychile/N?i=1039348\&f=2012-04-24.

23. Díaz R, Carrillo M, Terrazas J, Canales C. Actitudes que influyen en la demanda de 
servicios odontológicos durante la gestación. Rev ADM. 2001; 58(2): 68-73.

24. Ruiz León G, Gómez García R, Rodríguez Guerrero R. Relación entre la prevalencia de caries dental y embarazo. Rev ADM. 2002; 59(1): 5-9.

25. Carrión C. Diagnóstico de salud oral de primigestas que acuden a control prenatal al consultorio Dr. José Astaburuaga. Talca 2004 [Tesis]. Talca: Universidad de Talca, Facultad de Ciencias de la Salud. Escuela de Odontología, 2004; p. 45.

26. Díaz-Guzmán L, Castellanos-Suárez J. Lesions of the oral mucosa and periodontal disease behavior in pregnant patients. Med. Oral. Patol. Oral Cir. Bucal, 2004; 9(5): 434-7.

27. Artázcos J, Cortés F, Rosel E, Percepción y Hábitos de Salud bucodental en niños y adolescentes de Navarra. An Sist Sanit $\mathrm{Na}$ var., 2010; 33(1): 51-64.

28. González-Martínez F, Puello E, Díaz A. Representaciones sociales sobre la salud y enfermedad oral en familias de La Boquilla, Cartagena-Colombia. Rev Clin Med Fam., 2010; 3(1): 27-35.
29. Núñez J, Moya P, Monsalves MJ, Landaeta S. Nivel de Conocimiento de salud oral y utilización de GES odontológico en puérperas atendidas en una clínica privada. Santiago, Chile. Int J Odontostomat., 2013; 7(1): 39-46.

30. Pérez A, Betancourt M, Espeso N, Miranda M, González B. Caries dental asociada a factores de riesgo durante el embarazo. Rev Cubana Estomatol., 2011; 48(2): 104-112.

31. Montecinos C. Atención Odontológica en escolares: una evaluación pre y post intervención por odontólogo exclusivo en Chillán, Chile. Rev Cub Salud Pública [en prensa 2017].

32. Hernández B, Velasco-Mondragón H. Encuestas transversales. Salud Publ Mex., 2000; 42(5): 447-55.

33. Córdova J, Bulnes R. Nivel de conocimiento sobre prevención de salud bucal en gestantes, hospital de la mujer, Villahermosa, Tabasco 2007. Horizonte Sanitario, 2007; 6(2): 18-25. 